\title{
The Desiǵn of Model Propellers and Elastic Motors
}

\section{By E. A. Vessey}

THE science of model flying-machine making has been so fully dealt with through the medium both of separate articles in the various flight journals, and eve of small textbooks devoted to the subject, that it seem strange that the really useful information to be obtained concerning the correct design of propellers and motors to suit certain fixed requirements should be so meager The only article attempting to touch this branch of the subject which has come to the notice of the present writer is one by Mr. W. Langdon Davies on "Twisted writer is one by Mr. W. Langdon Davies on "Twisted Flastic Motors," in which he describes in detail some
very clever tests for ascertaining the maximum torque at a two-inch radius to be obtained from elastic skein of various proportions; and the maximum safe working twist. From these results he deduces the total work of the skein. The information contained in the article referred to is most useful; especially that relative to the safe amount of twist for the elastic, but unfortunately from the model maker's point of view it is no easy matter to apply such information to the actual design of a motor to generate the amount of power required in any given instance to accomplish flight ln scientific model making, as in the manufacture of the full-size flying machine, the question of propulsion resolves itself into the consideration of the thrus required and the velocity of flight, that is, it is first essentially a question of "power" pure and simple. For the sake of clearness it may be well to point out that the term "power" is used in its full mechanical that the term "power" is used in its full mechanical work being defined as "the product of the force exerted and the space passed over." Thus a model flying at 20 feet per second with a mean thrust of $1 / 2$ ounce will require to be provided with power represented by $1 / 2 \times 20=10$ foot-ounces per second.

If the model covers 300 feet before the elastic is un wound, the mean work done is represented by: $1 / 2 \times 300=150$ foot-ounces.

Now, Mr. Langdon Davies' figures give us only the work done; but say nothing as to the rate of doing it.

From these remarks it is not to be thought that the present writer wishes to detract in any way from the credit due to the earlier investigator. Such is not by any means the intention, the present article being framed rather as supplementary to Mr. Langdon Davies'; the threads being taken up where he left them.

There is reason to believe that the correct proportioning of the propellers and elastic motors of model to secure maximum effieiency is very little understood the reason being probably that everything is sacrificed to secure long flights.

The experiments about to be described were commenced many months since, with a view to finding, and if possible putting into some simple form, the laws governing the twisting and untwisting of elastic skeins. It was realized from the first that in order to secure useful results, the skein and propeller must be tested together, the power exerted by the skein being measured in terms of the thrust and absolute velocity measured in terms of the thrust and absolute velocity
produced when using propellers of varying proportions. The apparatus used will be described later. In comparing various skeins it was necessary, in order to insure that the results should be obtained under similar conditions, that the same relative twist should be given to each skein. It was found that the thrust given by a propeller driven by a twisted elastic skein did not, as might at first be expected, decrease gradually from a maximum to a minimum as the skein unwound, but fell in certain well-defined steps, remaining for relatively long periods at thrust values which were practically tively long periods at thrust values which were practically
constant throughout such periods. The "jump" in thrust was found to take place approximately when the skein was changing from one series of knots to another, the thrust being maintained practically constant throughout the unwinding of any one series of knots. This phenomenon accounts for the fact that a model in flight appears to lose its propulsive power suddenly, and not suddenly, and not gradually, as would at first seem rational. Since the thrust and velocity of the pro-
peller are both functions of the speed of rotation, it peller are both functions of the speed of rotation,
follows that the flight velocity also decreases in steps, follows that the flight velocity also decreases in steps, with the change from one series of knots in the skein to another. This being so, it was thought best for the purpose of the test to make the standard wind for all skeins not a certain number of turns, but a certain number of completed knots.

When a skein of elastic is wound up, it twists firs into a straight stranded rope, which gradually gets
tighter and tighter up to a certain point, when a further twisting causes a rapid decrease in the tension with the formation of a knot in the skein. Immediately after the formation of the first knot the tension in to secure reasonably high flights. of test. is to be cut,

$x=$ the thickness of ditto

$d=$ the diameter over propeller tips, Then:

$=$ the pitch angle at the blade tips,

$w=0.157 d$

and $t=0.157 d \tan \theta$ the skein again increases, and goes on increasing until the skein is uniformly knotted throughout its length when a second rapid drop in tension occurs and continues until the first of a second series of knots is formed. With the elastic dry or chalk-lubricated the second series of knots is incomplete when the fracture of the skein takes place; but if lubricated with one of the special .preparations provided by various makers, the complete series may be attained, and even part of a third series, though it is difficult to say exactly, by observation, when the second series ends and the by observation, when the second series ends and the
third begins. It seemed fairly obvious that the standard wind must be chosen so as to comprise a complete series of knots; and since a great many experimenter prefer to use the elastic untreated, the complete firs series of knots was decided upon, and various propellers were tested on standard skeins of varying numbers of strands, the skein always being wound to a complete series of first knots irrespective of the number of strands. In this way a good mean value for the thrust and absolute velocity was considered to be obtained, since obviously, whatever results are obtained by winding the skein, either lubricated or not, to its utmost safe capacity, the thrust and velocity for soaring must still be produced when the first series of knots is in operation, if any length of flight is desired. This method of design would appear to provide an ample surplus of power, when the skein is fully wound, to give the necessary acceleration after launching and

The elastic used in most of the tests was of the ordinary 1/16-inch square variety, this being chosen because it can be obtained in very long continuous lengths, also the section is practically uniform both throughout any one sample and in various samples. The strip elastic, as usually sold, is in comparatively short lengths, the width often varies along any one length, and it is difficult to find a series of lengths sufficient to form a skein which are of precisely the same widths. For a skein which are of precisely the same width. For
ordinary practical purposes this does not form a serious objection, but is prohibitive when making any form

The propellers were all cut from solid pine and polished. The working face of the blades was flat and of uniform pitch throughout its length. All propellers were made to standard proportions, which were arrived at after careful trial on actual flying models. The rule finally adopted as giving the best all-round results was:

If $w=$ the width of the block from which the propeller

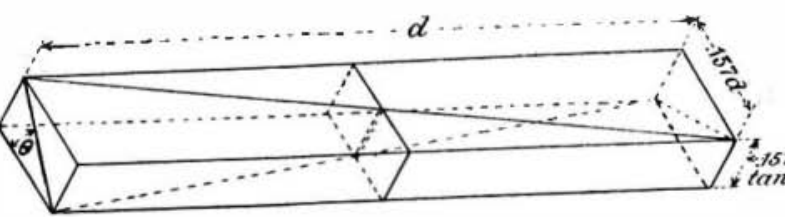

Fig. 1. The Propellor Marked Out for Cutting.

The profile of the blado was of modified Chauvière form with the leading edge curved and the trailing edge straight, the blade back being rounded. The length through the boss of the finished propeller measured along the axis of rotation was made $=\frac{t}{2}$, and the whole of the reduction was made from the trailing side.

Fig. 1 shows the propeller block marked out for utting, with the chief dimensions shown.

Fig. 2 shows the same block finished with the exception of shaping up to the standard profile, which is marked in dotted lines on the working face.

It will thus be seen that all propellers made to this rule have the same projected area, if the diameter is the same; the projected area varying directly as the square of the diameter on propellers of different diameters.

Such propellers were found to give excellent results under all conditions of practice, and could be run at very high speeds without any falling off in the proportional thrust.

The first test made was to find the law which governs the number of revolutions required to form a complete series of first knots in skeins of various lengths and numbers of strands. It was found that, within very narrow limits, the number of revolutions required is given by the expression:

$$
W=28.4 \frac{l}{N^{0.63}} .
$$

Where $W=$ the required number of revolutions

$l=$ the length of the skein in inches.

$N=$ the number of strands of $1 / 16$-inch square cord.

Table I gives the results for skeins 12 inches long, corresponding values for similar skeins of greater or less length being obtained by multiplying by the length in feet.

TABLE I.

\begin{tabular}{l|r|r|r|r|r|r|r|r|r|r|r}
\hline Number of strands. & 1 & 2 & 4 & 6 & 8 & 10 & 12 & 14 & 16 & 18 & 20 \\
\hline First knot revolu tions & 341 & 220 & 142 & 110 & 92 & 80 & 71 & 64 & 59 & 55 & 51
\end{tabular}

Since the formula gives the number of turns to complete the first series of knots, and since it would appear logisal to assume that fracture always occurs at proportional winds, the same formula should give the number of turns in the safe wind, if the constant be altered. It has been thought interesting to compare the results obtained by the equation thus adapted and the results obtained by the equation thus adapted and The comparison is therefore shown in Table II below:

TABLE II.

\begin{tabular}{|l|l|l|l|l|l|l|} 
No. of strands..... & 2 & 4 & 6 & 8 & 12 & 16
\end{tabular}

\begin{tabular}{ll|l|l|l|l|l} 
Langdon Davies. . & 402 & 283 & 234 & 203 & 156 & 143
\end{tabular}

$W=\frac{K}{N \cdot 63}$ \begin{tabular}{|l|l|l|l|l|l}
485 & 313 & 242 & 202 & 157 & 13 \\
\hline
\end{tabular}

The figures given by Mr. Langdon Davies are for skeins 12 inches long and are 75 per cent of the number of turns required to break the skein. In applying the formula it has therefore been necessary to fix a fresh value for factor $K$. For this purpose the wind for the 8-strand skein bas been taken as the mean value that the results agree fairly well. The discrepancy is that the results agree fairly well. The discrepancy is
probably explained by an error in the assumption that racture occurs in varying skeins at proportional winds; thick skeins apparently bearing more winding than thin ones. Small variations in the initial tension of the skein, and lubrication of the elastic, were found to have no appreciable effect on the above results.

The number of revolutions given by the above formula were adopted as the standard for each skein and used throughout the following tests.

A series of propellers was then made, varying in diameter from $51 / 2$ inches up to 12 inches and in pitch angle at the tips from about 19 degrees up to 35 degrees. The apparatus shown in Fig. 3 was then devised for measuring the thrust. The bar $A$, which carries the propeller bearing and anchor hook for the elastic, was made long enough to accommodate a 30-inch skein (this skin when relaxed was 24 inches long) and was supported so as to perit of its swinging by paralle links $B$ f from the rigid supports $C C$. Behind on of the links was fixed a scale reading in ounces and fractions the direct thrust on $A$. The scale was carefully calibrated by hanging known weights in a scale pan
suspended from the string $D$, which passed over the 
pulley $E$ and was attached to the end of the bar $A$ The pulley $E$ was made of large diameter and small bore to reduce friction effects to a minimum and was swung from a support $F$ (not shown) by a long link $G$. By this means the pull of the string was maintained parallel to the bar $A$ throughout a considerable swing and could be finally corrected by raising or lowering $F$. The aftermost link $B$ was continued above its support and shaped to accommodate a sliding weight $H$, which and shaped to accommodate a sliding weight $H$, which
could be fixed in any position. This was used to comcould be fixed in any position. This was used to com-
pensate for varying weights of elastic and propellers pensate for varying weights of elastic and propellers
when changing skeins, etc. The same link was provided with a lever $K$, more or less at right angles to it and projecting forward, from the outer end of which a long rod led downward to a dash-pot with a moderate damping effect to make the readings as dead beat as possible. To avoid all possible chances of error, the calibration of the scale was repeated on every change of skein and propeller, after the compensating change of skein and propeller, after the compensating
weight $H$ had been set. The scale pan and string were left attached throughout the tests. The propeller spindles were mounted each on a plain bearing, and the thrust was taken on two small flat washers. The duration of the run with each combination of skein and propeller was taken with a stop-wateh. The skeins, as has already been said, were invariably wound to a complete set of first knots, and readings were taken for time and thrust for each combination. Three sets of readings were taken in each instance, and the mean values adopted as correct. Readings were also taken with various lengths of skein, from which it was found that, whatever the length of skein, the thrust and velocity remained constant, but the time varied in direct proportion to the length of skein. It was also noted that, whether the skein was run dry or lubricated, the values of the variables remained unaltered: but there is reason to believe that, after the lubricant has thoroughly permeated the rubber, it. has the effect of considerably diminishing all the values. Various sections of rubber were tried, and it was found that, so long as the total area of section remained constan the results were practically unaffected.

The effect of reducing the area of the blade within small limits was tried, and it was found that, provided the diameter of the propeller was kept constant, no appreciable effect resulted. A considerable reduction in the width of blade at the root was also made without appreciable effect.

In this way a number of sets of comparative data were found. The results were then plotted and the mean curve drawn. An equation was then found for each set of variables which satisfied the conditions within the limits of practice. The resulting equations were as follows:

$$
\begin{aligned}
& T=\frac{19 N}{29-N} \cdot d^{-4}(\tan t) \\
& V=3910 \frac{N^{.37}}{d^{1 \cdot 5}(40-N)} . \\
& \iota=\frac{40-N}{770 N} \cdot d^{2.5} l \cdot \tan \prime
\end{aligned}
$$

and fing arrived at are somewhat alarming in aspect, owing to the fractional indices. Although the solutions involve the use of eomparatively simple mathematics, still there is no doubt the manipulation may be beyond the capacity of many enthusiastic investigators, especially the more juvenile. It has therefore been thought advisable to present the result arrived at in the form of a simple chart, by the use of which all mathematical work is climinated and the solution becomes merely a process of eorrectly following certain curves.

The chart is given in Fig. 4, and will be seen to consist of two separate sections. The upper section is ing from 3 inches $t 0$ is inches, and a horizontal seale of mean absolute velocity from 1 to 40 miles per hour. The diagonal lines, sloping upward toward the right hand, show the relation between the thrust and diameter for varying tip angles, and those sloping upward toward the left the relation between the velocity and diameter for varying numbers of strands in the skein. In the lower section the vertical seale gives the number of lower section the vertical scale gives the number of mum thrust in ounces. The curves show the relation mum thrust in ounces. The curves show the relation
between these two factors. To explain the use of the between these two factors. To explain the use of the
chart, we will suppose that we have a propeller of chart, we will suppose that we have a propeller of 8-inch diameter with a tip angle of 26.5 degrees, and we require to know what thrust and velocity it will give us on a 10-strand skein. (N. B.-The skein length makes no difference.) In passing, it must be pointed out that there are of course an infinite number of diagonals; a few only being drawn for the sake of clearness. The a few only being drawn for the sake of clearness. The
others may be imagined as lying parallel to those drawn, and their position may be located by proportionate division. Thus, the diagonal for a tip angle of 26.5 degrees will be approximately at a quarter of the distance between diagonals marked 26 degrees and 28 degrees, and measured from the former. Having found 8 inches, the given diameter, on the left-hand vertieal seale of the upper section, we trace horizontally until we meet the imaginary we meet the imaginary and then vertically downward to the dividing angle, and then vertically downward to the dividing line between the two sections. The course is shown
in dotted lines. If our skein had consisted of one strand in dotted lines. If our skein had consisted of one strand
we should have traced on vertically downward, emerging at 0.09 ounce thrust, but the skein has 10 strands, and correction is made for this condition by tracing diagonally parallel to the nearest curve until we meet the horizontal marked 10 on the left-hand scale of the lower section, and then vertically downward, emerging lower section, and then vertically downward, emerging
at a thrust of 1.26 ounces, which is the required thrust. To find the velocity, we go back to the upper section, and find that the horizontal dotted trace from 8-inch diameter has already intersected a diagonal sloping upward to the left and marked 10 strands. From this intersection we trace vertically upward, emerging at 13.3 miles per hour, which is the velocity required. This is called the mean absolute velocity, but the actual mean flight velocity may be taken safely as about 10 miles per hour. It is interesting to note that this 10 miles per hour. It is interesting to note that this
identical propeller on test gave $11 / 4$ ounces thrust and identical propeller on test gave $11 / 4$ ounces thrust and
13 miles per hour mean absolute velocity. It is of 13 miles per hour mean absolute velocity. It is of
course hardly necessary to point out that all propellers treated by means of this chart must be of the proportions set out earlier in the article and illustrated in Figs. 1 and 2

Suppose now we have a model which is eapable of sustaining a total weight of 2 ounces, at which weight it glides in still air at an angle of about 1 in 6 , traversing a distance of about 35 feet in $2 \frac{1}{2}$ seconds. Its natural speed is thus $\frac{35}{2 \frac{1}{2}}=14$ feet per second, or about 9.5 miles per hour. For this machine we wish to design the motor and propeller. The gliding angle being 1 in 6 , the actual thrust required for soaring will be $2 / 6=1 / 3$ ounce; and since our chart is hased on the elastie being wound only to complete the first full series of being wound only to complete the first full series of
knots, we do not require to make much allowance over and above this to cover emergeneies (such as acceleration and rising) since this is automatically provided when the skein is fully wound. If the elastic is used dry and wound to the Langdon Davies standard

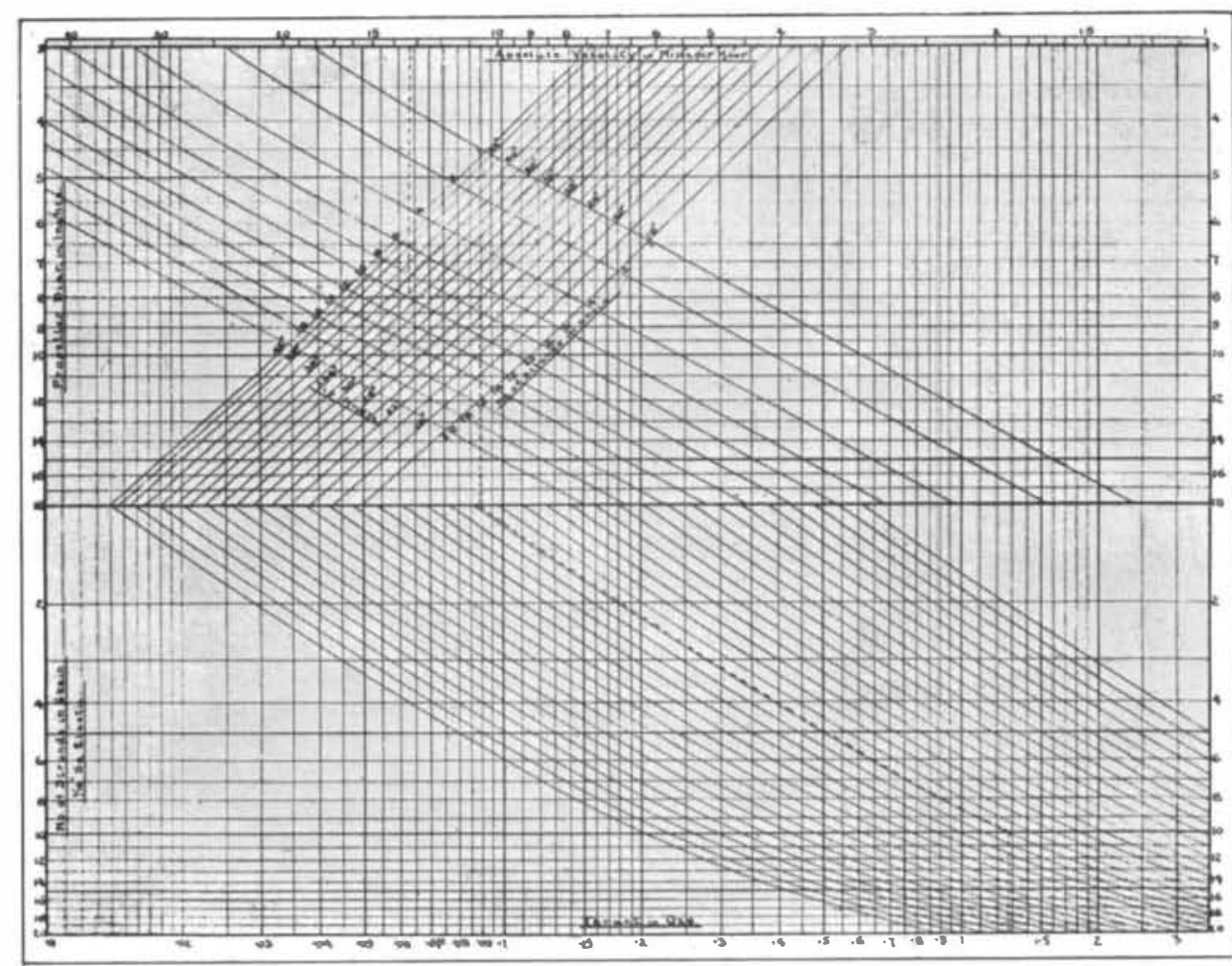

Fig. 4. Chart sloowing the Relition lietween the Numler of strands in slisein and the Maximum 'Thrust Oltainable.

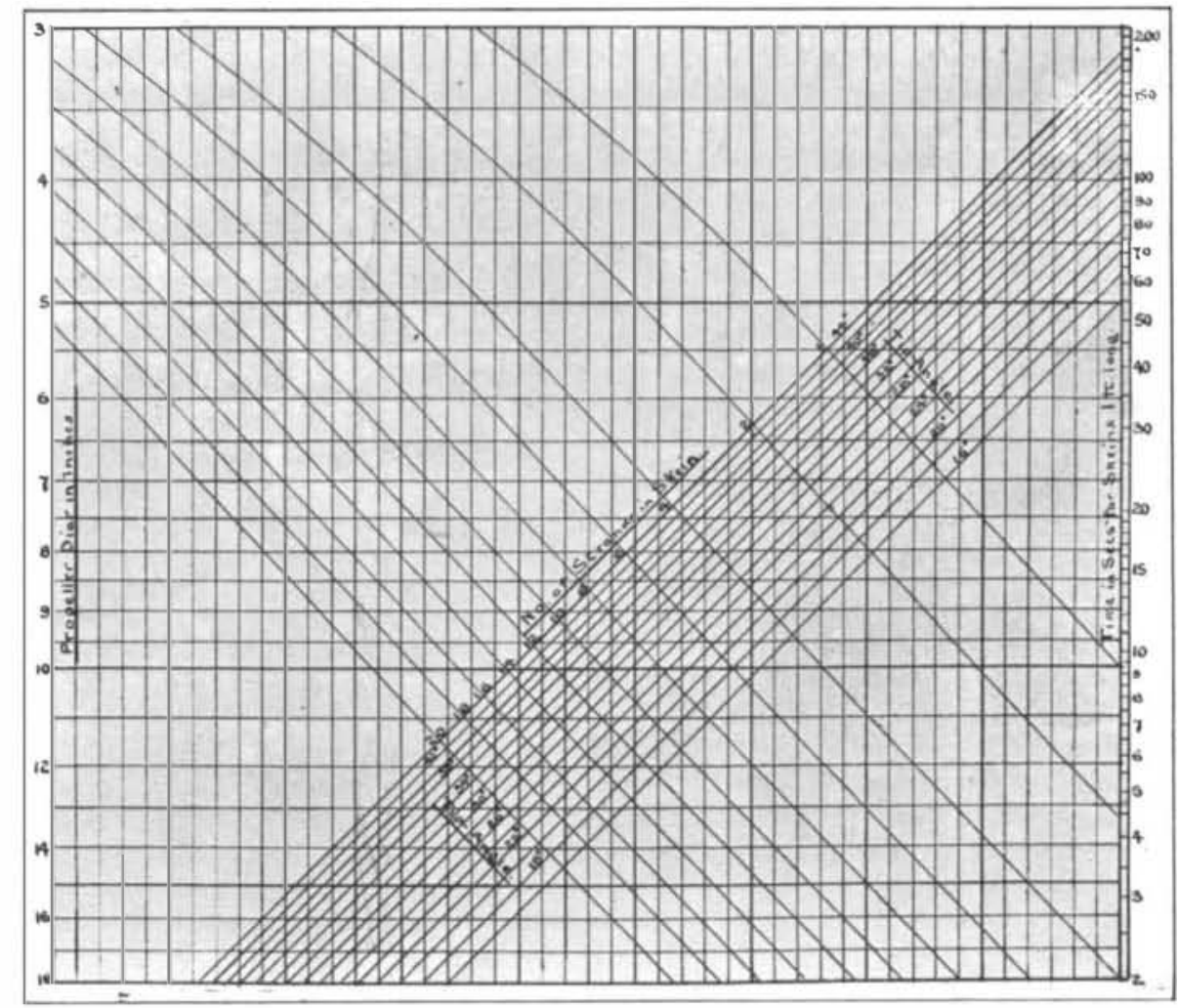

Fig. 5. Chart Showing Time Taken by Various skeins in Lnwinding. 
tho maximum thrush would be about twicu and a cyartor that given by the chart, the maximum velocity being about 1.5 times the charted value. In the present case it will be wise to allow for say $1 / 2$-ounce thrust on the full skein of first knots. Allowing about 30 per cent slip, we find the mean absolute velocity to be consult the chart as to all possible combinations which give the desired results. It must be borne in mind that the allowances made above are purely arbitrary, that the allowances made above are purely arbitrary, being results of the writer's personal experience. The
slip and added thrust may obviously be altered to suit the designer's individual taste and requirements without affecting the argument in any way. Referring to the chart, let us commence with the smallest number of strands, viz., 2. Starting from 13.5 on the velocity scale, we trace vertically downward to intersect with the 2-strand diagonal. This intersection takes place scale. This, therefore, is the diameter for the propeller to give the desired velocity with two strands of elastic. Let us now see what tip angle we must give it to obtain 0.5-ounce thrust. Starting from 0.5 on the thrust scale on the bottom of the chart, we trace vertically upward to intersect with the horizontal from 2 on the "strands" scale of the lower section, and then from this intersection upward again but parallel to the diagonal curves until we reach the division line of the chart. Thence we trace vertically until we intersect chart. Thence we trace vertically until we intersect
with the horizontal from the diameter already found, viz., 4.625 inches. Through this intersection a tipangle diagonal will either actually pass or may be imagined. If it is imaginary, its value may be judged by its position relative to the diagonal on either side of it. In the present instance the value is $201 / 2$ degrees. Our propeller is therefore $45 / 8$-inch diameter with a tip-angle of $201 / 2$ degrees. By a similar process of
tracing we find propellers for other skeins. Thus: racing we find propellers for other skeins. Thus:
A 4-strand skein requires a 5 5/8-inch propeller with $32 \frac{1}{2}$ degrees tip-angle.

A 6-strand skein requires a $61 / 2$-inch propeller with 40 degrees tip-angle.

This is the largest skein we can use, since with 8 strands the tip-angle becomes greater than 45 degrees, which is inadvisable. Let us assume that we can accommodate a skein 30 inches long. In Fig. 5 is given a chart which shows the time taken by various skeins in unwinding, when driving various propellers. The left-hand scale gives the propeller diameter in inches, left-hand scate gives the propeller diameter in inches,
the right-hand scale the time in seconds during which the completely single-knotted skein 12 inches long unwinds. The diagonals sloping upward to the left refer to the number of strands in the skeins, and those sloping upward to the right, to the tip-angle in degrees. From the chart let us now find out how long each of the above propellers may be expected to run. Taking the smallest diameter first, we find 4.625 on the leftthand scale, and trace horizontally to intersect the skein diagonal marked 2. From this junction we proceed vertically downward to intersect the tip-angle diagonal marked $201 / 2$ degrees, and thence horizontally to the right to $53 / 8$ seconds on the time scale. This is the time for a single-knotted 12-inch skein of two strands; and since the time varies directly as the skein length, it becomes for a 30-inch skein $53 / 8 \times 30 / 12=131 / 2$ seconds. If the skein is wound up to the Langdon
Davies standard for unlubricated elastic, the duration Davies standard for unlubricated elastic, the duration
of the run is increased by 50 per cent over the charted values. Hence the time of unwinding of the $45 / 8$ inch $\times 201 / 2$-degree $\times 2$ strand combination is about 20 seconds. Similarly, the times for the $55 / 8$-inch $\times$
$321 / 2 \times 4$-strand, and $61 / 2$-inch $\times 40$-degree $\times 6$-strand combinations are respectively 27 and 32 seconds.

Now, these are all combinations of equal power, since they give practically the same thrust and velocity in each case. The power-to-weight ratio is given by the expression $\frac{T \times V}{\text { No. of strands }}$ for skeins varying only as regards number. of strands, where $T=$ thrust, and $V=$ velocity.

Therefore, comparing the three examples above, we find the power-to-weight ratio to be:

(1) $\begin{aligned} \frac{T \times V}{2}=\frac{.5 \times 13.5}{2}=\frac{6.75}{2} & =3.375 \\ \text { (2) } & \frac{6.75}{4}=1.6875 \\ \text { (3) } & \frac{6.75}{6}=1.125\end{aligned}$

Whence combination No. 1 is the best. If, however, we desire to secure the greatest duration of flight, we must use combination No. 3 . Similarly, since the
velocity is the same throughout the series, No. 3 will velocity is the same throughout the series, No. 3 will
give the longest flight, provided skeins of the same length give the longest flight, provided skeins of the same length
are used in each instance. Suppose, however, for the are used in each instance. Suppose, however, for the of the first two skeins equal to that of the third by lengthening them; thus, making No. 1 skein 90 inches long, and No. 2, 45 inches long. How do the distance
and duration values now compare with No. 3 combina- lion? Since tlo time is directly pruportional to tho length of skein, the new 2-strand skein will now run 60 seconds, and the new 4-strand skein 40 seconds, which shows that, weight for weight, long skeins of few strands ar to be preferred to short skeins of many strands, provided always that propellers to suit are provided. It must not be thought that the writer is hereby recommending a skein of 90 inches long for a 2-ounce model; that of course is impracticable. The point he wishes that of course is impracticable. The point he wishes practice in design to use the longest skeins possible with the fewest strands and small propellers. With the first assertion all model makers will probably agree, but as regards the other two there will, without doubt, be some difference of opinion. In ordinary full-size theory, everything points to the advisability of using large propellers of high pitch ratio, yet how very seldom are such propellers seen in practice. The question of weight in the prime mover and transmission sets the weight in the prime mover and transmission sets the
limit. The only way as yet discovered to keep the motor weight down is to run it at a high speed, which of course necessitates the use of a propeller of relatively small pitch and diameter, with corresponding loss in efficiency. Geared propellers have been used, but the unavoidable complication involved in their employment seriously discounts the gain in efficiency in the propeller itself. It therefore is evident that the point at present to be considered in full-sized design is, by force of circumstances, expediency rather than efficiency although it is not for a moment suggested that genera all-round efficiency should be ignored. As with the full-sized machine, so with the model. To obtai the best results with the materials at hand, we must seek the best combination; and, as has been shown, such does not necessarily include the most efficient propeller. The writer's aim is to secure the best all-round combination, which, in his opinion, is such as will give the longest reasonable flight both as regards distance and duration with the smallest motor, i. e., th least amount of elastic. It is admitted that it is quit possible to select one item in the combination and show that it is not theoretically most efficient, just as has been shown to be the case with the full-sized machine but the whole of the tests go to prove that the happy medium aimed at has been found.

Now, just a word as to efficiency and "slip." Effciency is defined as the ratio between the useful horsepower (which is directly proportional to the product of the thrust and the velocity forward of the propeller) and the actual brake horse-power of the motor. Treating the latter first, the power of an elastic motor is
a very variable quantity, since it depends, all other things being equal, on the rate at which it unwinds. This may easily be realized when it is remembered that in winding up a certain skein a given number of turns a certain fixed amount of work is done, and this amount of work, with certain very small deductions for friction losses, will be delivered up again by the skein in untwisting. Now "power," as has been already stated, is the "rate of doing work;" hence it is not difficult to see that power depends entirely on the speed of untwisting. In other words, an elastic motor to give maximum power with minimum weight must necessarily unwind at a high speed. This bein comprises a relatively small propeller, is amply borne comprises a relatively small propeller, is amply borne out, since a precisely similar line has to be followed in
full-sized design when dealing with high-speed gasoline motors. Suppose that the work given out by the skein in untwisting is represented by $W$ and the time take is called $t$, then neglecting friction losses the brake $=\frac{u^{*}}{t}$. For instance, say the skein is capable of performing, say, 500 foot ounces of work. For the sake of argument, say it completely unwinds in 10 seconds, when a certain propeller is used; the power is therefore $500 / 10=50$ foot ounces per second. But supposin this propeller is changed for another which only allows of unwinding in say 60 seconds, then the power or rate at which the work is performed is $500 / 60=8.3$ foo at which the work is performed is $500 / 60=8.3$ foot
ounces per second only. We may, therefore, increase ounces per second only. We may, therefore, increas
the power of our skein by putting faster-running propellers to it, and vice versa. So much for the hrake power; now as to the useful power. This is propor-
tional to the product $T \times V$ where $T=$ thrust and tional to the product $T \times V$ where $T=$ thrust and
$V=$ velocity of flight. The efficiency is, therefore given by the ratio:

$$
\frac{\text { Useful power }}{\text { brake power }} \text { which equals } k \cdot \frac{T V t}{W}
$$

Where $k$ is a constant. Since our object is to find the most efficient propeller for a given number of strands of elastic, we may assume $W$ as constant. The expression therefore becomes: Efficion

Substituting for the various factors from equation (2), (3), and (4) and remembering that in all these
equations $N$ is constant, we obtain: Efficiency $=$ $K_{1} \frac{l}{\sqrt[3]{d}}=\left(\right.$ where $K_{1}$ is a constant including $\left.N\right)$. Whence wo soo that tip-angle has no effect on the efficiency, and that a long skein and small propeller are imperative if high efficiency is desired.

In the above investigation one correction is necessary $V$ in the formulø should be the actual velocity of flight, and not, as we have made it, by substituting the value make this corrotion we must make a certain deduction make this correction we must make a certain deduction slip is a variable quantity, $i$. e., it is not only different for different propellers, but varies for any given propeller under differing conditions of load. As far as the writer is aware, no reliable means of calculating this quantity have at present been published, though tests are now being made with propellers under flight conditions with a view to establishing the necessary data.
As the matter stands at present, the only course appears to be to assume a safe value, and 30 ner cent appears to be to assume a safe value, and 30 ner cent appears ing this value, the expression for efficiency becomes

$$
\text { Efficiency }=\cdot 7 K_{1} \frac{l}{\sqrt[3]{d}}=
$$

It must be pointed out that the inclusion of this slip allowance in no way affects the general argument, since it merely alters the constant $K_{1}$.

As has already been said, the above argument proves that the tip angle of the blade has no effect on the efficiency of the propeller when driven by an elastic motor; consequently, in designing we should naturally select a fairly steep angle in order to secure the requisite velocity with a small propeller diameter, and also maximum duration of flight and distance. A pitch ratio of $3: 1$ will give us a tip-angle of about $431 / 2$ degrees, which is in the writer's opinion larger than is advisable.
Better results are obtained by using a maximum pitch ratio of $2.5: 1$, which requires a tip-angle of $381 / 2$ degrees, though a larger angle may of course be used in exceptional cases. Having decided on the angle, it is very easy from the chart to settle the diameter and number of strands required to secure the velocity and thrust called for by the model. The propeller must then be made to the proportions set down earlier in the article. In conclusion, the writer would like to state that the not claimed as being the "one and only" way, but propellers so designed have been found to give excellent results in practice; and further, it is, so far as is personally known, the only detailed method of design yet published and as such, should prove of interest and use both to those uninitiated in the mysteries of the theory of Aeronautics, and it is hoped also to their more technical brethren.

\section{Oil in Boilers}

"When the boiler-feed water is taken from the hotwell of a jet condenser," "says Power," "as is the practice where fresh water is available for condensing there is little danger that excessive quantities of oil will be carried to the boiler. There is believed to be little danger to any type of boiler from the oil itself because,
being lighter than the water, it will not sink to the bottom and become attached to the plates. The error in this helief lies in the assumption that what is oil when it. enters the boiler remains oil. It does not. Subjected to the long continued high temperature of the water, it is slowly distilled, that portion which is volatilized going off slowly distilled, that portion which is volatilized going off
with the steam, leaving behind a residue of a higher specific gravity than water, which sinks to the bottom as soon as the circulation stops. When cold it is firm and non-adhesive, but when heated is not unlike asphaltum. "So long as the circulation continues, this matter will travel along with the current and do no harm. adheres to it so strongly that it is not dișlodged when the circulation starts again. Water is kept- from contact with the sheet and a 'bag' is the result.

"When a bag is caused by oil it is liable to be anywhere on the shell below the fire line. It is usually near
the bottom, but may be well up on the side and anywhere between the front and rear heads.

"The mass that clings to the sheet is not adways entirely oil residual. It is often found agglomerater with the scale-making solids of the water in irregular masses in all parts of the boiler.

"What is really more dangerous, beanuse less tiablo to be suspected, is the milky-colored emulsion which goes to the boiler after the heavier-bodied oil has been removed by filtration. This does not behave like oil proper in associating with foreign matter nor by gathering in large or small masses, but goes atom by atom to hard, bright, black varnish, which as effectually prevents the contact of water as the thickest coat of scale. It covers the entire sheet below the fire line before it is noticed and the 'bag' is often as wide as the diameter noticed and the 'bag' is often as wide as the diameter
of the boiler and as long as the sheet. This bagging is gradual, as at the first slight stretch the varnish cracks and the water cools the softened sheet and restores its strength until the coat of varnish again co"ers it; this
is repeated until the sheets are bulged like a barrel." 